\title{
PAPILOMAS INVERTIDOS - CASUÍSTICA DEL SERVICIO Y REVISIÓN DE LA LITERATURA
}

\author{
L. XAMBRE*, R. PRISCO*, F. CARREIRA*, M. HONAVAR**, R. LAGES* \\ *Servicio de Urología. **Servicio de Anatomía Patológica. Hospital Pedro Hispano. Matosinhos. Portugal.
}

Actas Urol Esp. 27 (8): 605-610, 2003

\section{RESUMEN}

\section{PAPILOMAS INVERTIDOS - CASUÍSTICA DEL SERVICIO Y REVISIÓN DE LA LITERATURA}

Los papilomas invertidos son neoformaciones raras, responsables de aproximadamente $2,2 \%$ de las neoplasias uroteliales (benignas o malignas). Son generalmente localizadas a nivel vesical, aunque existen casos descritos de otras localizaciones en el sistema excretor urinario.

Los autores presentan seis casos de esta variedad de neoformación, señalando las características clínicas de cada uno, su forma de presentación, características de las neoplasias, actitudes terapéuticas y su evolución. Es realizada también una revisión de la literatura, sobre la clínica, criterios de diagnostico, tratamiento y pronóstico.

PALABRAS CLAVE: Tumor urotelial. Papiloma invertido.

\section{ABSTRACT}

\section{INVERTED PAPILLOMAS - OUR SERVICE EXPERIENCE AND A REVIEW OF LITERATURE}

Inverted papillomas are rare tumours representing about $2.2 \%$ of all urothelial tumours (benign or malignant). Inverted papillomas are predominantly seen in the bladder but these tumours have also been described in other locations, all over the urinary tract.

The authors report six additional cases of such tumours describing the individual characteristics of each case, presenting symptoms, treatment employed and follow up available. A review of the literature relative to aetiology, clinical signs and symptoms, diagnostic criteria, treatment options and outcome has also been carried out.

KEY WORDS: Urothelial tumour. Inverted papilloma.

L os papilomas invertidos son lesiones uroteliales poco frecuentes, responsables por cerca del 2,2\% de las neoplasias uroteliales (benignas y malignas) ${ }^{1}$. Esta lesión pasó a ser llamada de esta manera y reconocida como entidad patológica autónoma desde que Potts y Hirst la describieron en $1963^{2}$ aunque se acepta que existe una comunicación previa que hace referencia a esta entidad, realizada por Paschkis en $1927^{3}$.
Presentan una localización mayoritariamente vesical, aunque se han descrito en otras localizaciones en el sistema urinario, tales como pelvis renal, unión pieloureteral, ureter o incluso en la uretra anterior ${ }^{4}$. Hasta el momento presente aparecen relatados en la literatura aproximadamente 300 casos, la mayoría bajo la forma de casos aislados o en pequeñas series. El diagnóstico es histológico y está basado en criterios bien definidos ${ }^{5}$. 
Presentamos seis nuevos casos de papilomas invertidos, cinco referentes a papilomas vesicales y uno de localización uretral (Tabla I).

\section{CASOS CLÍNICOS}

Caso 1

Paciente del sexo masculino, 59 anos, metalúrgico, sin antecedentes personales de interés. Refiere múltiples episodios de hematúria total con salida de coágulos desde aproximadamente tres meses antes. Niega quejas urinarias bajas. Niega hábitos tabaquicos. Realizó UIV que mostró defecto de substracción a nivel del suelo vesical, sin otras anomalías. Sometido a RTU. Se observó neoformación de aproximadamente $20 \mathrm{~mm}$ de diámetro en la pared vesical posterior.

Diagnóstico histológico: Papiloma invertido

Después de 8 años de control médico no fué detectada recidiva de la lesión o neoplasia urotelial metacrónica.

\section{Caso 2}

Paciente del sexo masculino, con 72 años, seguido en la consulta de urología por quejas urinarias bajas de tipo obstructivo, con varios años de evolución. Refería cuadro de hematuria macroscópica abundante con abundantes coágulos, aproximadamente 5 días antes. Antecedentes: hábitos tabaquicos, coronariopatía y bloqueo de rama miocárdico.

Ecografia: neoformación localizada en la base de la pared izquierda, con aproximadamente 2,5 $\mathrm{cm}$.

Cistoscopia: localizada en la región retrotrigonal, del lado izquierdo, neoformación de aspecto pediculado, sangrativa, la cual fué resecada. Histología: papiloma invertido.
Aproximadamente tres meses después tiene nuevo episodio de hematuria.

Ecografia: neoformaciones vesicales multifocales. Fue sometido a RTU. El estudio histológico de las piezas demostró neoplasia vesical con infiltración de la capa muscular. Sometido a cistoprostatectomia radical con linfadenectomia iliacoobturadora y ureterostomia transileal. El estudio histológico de la pieza operatoria confirma el diagnostico de neoplasia urotelial invasiva.

\section{Caso 3}

Paciente del sexo masculino que entró en el Servicio de Urgencia presentando hematuria macroscópica con emisión de coágulos de quince días de evolución. No refería quejas bajas. Fumador. Sin otros antecedentes de interés. Realizó ecografia la cual demostró una voluminosa masa intravesical heterogénea, móvil, sugestiva de coagulo. Sometido a RTU, fueron evacuados numerosos coágulos, siendo posible ver una pequeña neoformación ligeramente elevada localizada en el suelo vesical, de cerca de 5 $\mathrm{mm}$, la cual fué resecada. Histologia: papiloma invertido.

Después de tres meses de follow up, no hubo evidencia de recidiva o carcinoma urotelial.

\section{Caso 4}

Paciente del sexo masculino, 57 años de edad, quién refiere quejas urinarias obstructivas e irritativas moderadas. Niega antecedentes de hematuria macroscópica. Niega hábitos tabaquicos. Antecedentes de tuberculosis pulmonar. Realizó ecografia vésico-prostática la cual mostró una formación polipóide con aproximadamente $10 \mathrm{~mm}$.

\section{TABLA I}

CARACTERÍSTICAS DEMOGRÁFICAS Y CLÍNICAS

\begin{tabular}{||c|c|c|c|c|c|c||}
\hline Caso & Edad & Sexo & Localización & Presentación & Seguimiento & Recidiva (S/ N) / CCT (S/ N) \\
\hline 1 & 59 & M & Vesical & Hematuria & 96 & N/N \\
\hline 2 & 72 & M & Vesical & Hematuria & 108 & N/S (CCT invasivo) \\
\hline 3 & 57 & M & Vesical & Hematuria & 3 & N/N \\
\hline 4 & 57 & M & Vesical & Hallazgo ecográfico & 36 & N/N \\
\hline 5 & 14 & F & Vesical & Hallazgo ecográfico & 14 & N/N \\
\hline 6 & 54 & M & Uretra prostática & Hallazgo histológico & 50 & N/N \\
\hline
\end{tabular}


Sometido a RTU, fue confirmada la presencia de neoformación vesical polipóide sesil en la transición del suelo vesical con la pared izquierda.

Histologia: papiloma urotelial de tipo invertido acompañado de lesiones exuberantes de cistitis glandular.

Después de 36 meses de follow up regular, no hay evidencia de recurrencia o señales de carcinoma urotelial.

\section{Caso 5}

Paciente de 14 años de edad, seguida en la consulta de ginecología por quejas de dismenorrea. Realizó ecografia pélvica que puso en evidencia una formación polipóide del suelo vesical. Negaba cualquier queja urinaria baja o episodios de hematúria macroscópica. Antecedentes personales irrelevantes. Examen objetivo dentro de la normalidad.

La ecografia mostró una formación polipóide con aproximadamente $9 \mathrm{~mm}$ en el área de la pared izquierda.

Sometida a RTU en la cual se encontró neoformacion pediculada con las dimensiones referidas, en la transición del suelo con la pared izquierda vesical. La histologia puso en evidencia un papiloma invertido de tipo trabecular.

Hasta el momento (después de 14 meses de seguimiento) no hay registro de recidivas o evidencia de carcinoma de células de transición.

\section{Caso 6}

Paciente de 54 años de edad, sexo masculino, seguido en la consulta de urología por LUTS, mayoritariamente de tipo obstructivo. Negaba episodios previos de hematúria macroscópica.

Sometido a RTU de próstata, el estudio histológico mostró papiloma invertido de la uretra prostática.

Después de 50 meses de seguimiento se mantiene asintomático y no se verificó recidiva o aparecimiento de carcinoma urotelial.

\section{DISCUSIÓN}

El término papiloma invertido fue usado para describir una neoformación vesical por primera vez en 1963 por Potts y Hirst, pero de acuerdo con Anderstrom fue Paschkis quien describió inicialmente la lesión, describiendo cuatro casos que denominó como tumores adenomatosos vesicales, presentando las características hoy aceptadas para el diagnóstico de estas lesiones ${ }^{6}$.

Se trata de una entidad patológica rara, estando descritos en la literatura hasta el momento cerca de tres centenas de casos. En una série de 1.829 casos de tumores uroteliales clasificada por Kunze, apenas 2,2\% correspondían a papilomas invertidos ${ }^{1,7}$. En todas las series publicadas se verifica un predominio de pacientes del sexo masculino cuando comparado al femenino, variando entre $3: 1$ a $7: 1$, con un valor medio de $5: 1^{8}$, lo que contrasta con el carcinoma urotelial, el cual presenta una relación situada en 2:1. En nuestra casuística se confirma esta misma observación, siendo encontrada una relación de 5:1.

Habitualmente surgen en pacientes en la sexta o séptima década de vida, pero pueden aparecer en cualquier edad, existiendo mismo casos descritos en niños ${ }^{16}$. De cualquier manera, en las mayores series publicadas, la gran mayoria de los pacientes presentaban edades comprendidas entre los 50 y los 79 años. La edad média en la casuística presentada fue de 52 años, lo que está de acuerdo con las séries publicadas por otros autores. Es de señalar el hecho de que uno de los casos tiene características particulares, una vez que ocurre en un grupo etario poco frecuente.

La mayoria de los casos descritos (cerca de 90\%) corresponden a localización vesical, sobretodo a nivel del trígono, cuello vesical y uretra prostática. Hay no en tanto casos localizados virtualmente en todas las posibles localizaciones a lo largo del árbol excretor urinario. Las lesiones del aparato excretor alto corresponden sensiblemente a cerca de $3 \%$ del total. Normalmente se trata de lesiones únicas con dimensiones modestas, comprendidas entre los 5 y los $30 \mathrm{~mm}$. La localización multifocal está descrita en menos de $10 \%$ de los $\operatorname{casos}^{4}$. En la casuística del Servicio, cuatro de los casos apurados corresponden a neoformaciones vesicales (cuatro de las cuales situadas en el suelo y una en la pared vesical izquierda). En uno de los casos la neoformación estaba localizada en la uretra prostática. No tenemos registro de ningún caso de papiloma invertido del excretor alto.

Esta neoformacion normalmente se manifiesta por hematuria macroscópica o síntomas uri- 
narios bajos de tipo obstructivo, especialmente en los casos de lesiones localizadas a nivel del trígono, cuello vesical o uretra prostática. En muchas de estas situaciones, las quejas urinárias obstructivas son probablemente el resultado de hipertofia prostática concomitante y no resultado de la presencia del propio papiloma ${ }^{8,9}$. En los casos de lesiones con localización ureteral, pueden manifestarse con lumbalgia, aunque sea mas frecuente su presentación con hematuria. Pueden igualmente cursar de forma asintomatica. Estas características clínicas están patentes en la forma de presentación de los casos descritos. Encontramos la hematúria macroscópica como forma inicial de presentación en tres casos. Dos de los casos cursaron de forma perfectamente asintomatica, siendo hallazgos ecográficos. En un caso adicional el paciente presentaba síntomas obstructivos, muy probablemente resultado de hipertrofia prostática asociada, visto que cuando realizada la RTU de la próstata no fue visible cualquier neoformación con las características abajo referidas en la loca prostática. El diagnóstico en este caso fue hecho de forma retrospectiva, durante el examen anatomopatológico de las piezas obtenidas durante la intervención quirúrgica.

Los hallazgos radiológicos son perfectamente inespecíficos, encontrándose en ocasiones defectos de substracción intraluminal (30 a 50\% de los casos) $)^{8}$ o aspectos compatibles con obstrucción infravesical, mas una vez ocasionados por patología concomitante. En otras situaciones los estudios son perfectamente normales. Los hallazgos ecográficos son igualmente inespecíficos e indistinguibles de los encontrados en los carcinomas uroteliales (Fig. 1).

Tal como para todas las neoplasias vesicales, la endoscopia constituye el método diagnóstico de elección. Estos tumores normalmente aparecen como pequeños nódulos elevados, sólidos o por veces pediculados, con una base de implantación larga, de superficie lisa o mamelonada, con la localización y dimensiones ya referidas. El aspecto papilar (propio de los carcinomas de células de transición) no es característico ya que el crecimiento se realiza de forma endofítica y el epitelio que los recubre es de aspecto característicamente normal.

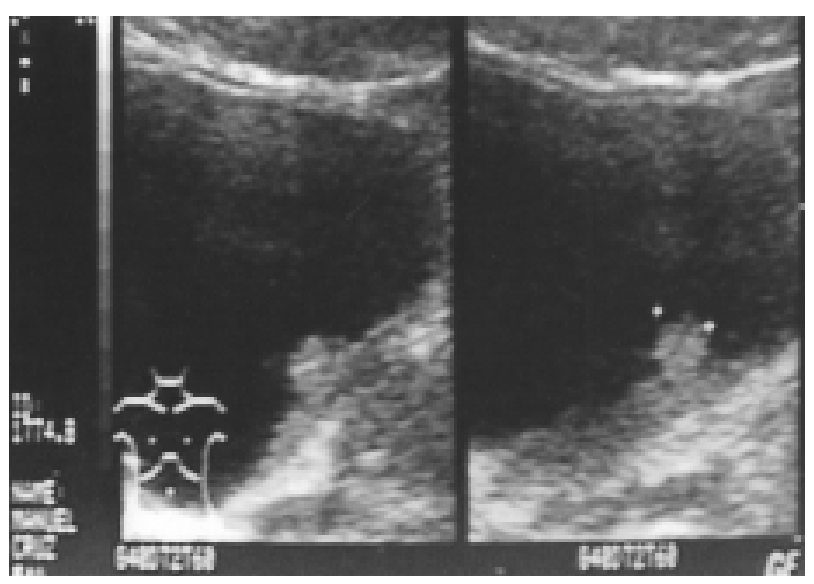

FIGURA 1. Papiloma invertido -hallazgos ecográficos.

Una vez que los papilomas invertidos son recubiertos por epitelio de transición normal y que su crecimiento se realiza en el sentido intramural, la citología urinaria carece de valor diagnóstico. La morfología citológica es en la mayoria de las situaciones normal o con alteraciones reactivas inespecíficas. Los pocos registros de citologías positivas corresponden a situaciones de tumores uroteliales síncronos, que son relativamente frecuentes como veremos.

El diagnóstico es en ultima instancia anatomopatológico, fundamentado en criterios histológicos específicos descritos por Henderson ${ }^{5}$ (Tabla II). Los hallazgos típicos son los de una lesión polipoide recubierta por epitelio transicional normal. Por bajo de esta superficie se observan numerosas trabeculas, cordones o macizos celulares comunicantes e interdigitantes, envueltas por un estroma de tejido conectivo laxo (Figs. 2 y 3). Las células presentan un patrón monomórfico, mitosis raras o ausentes, dando origen a un aspecto de empali-

\section{TABLA II}

CRITERIOS HISTOLÓGICOS DE DIAGNÓSTICO DE HENDERSON

Criterios de diagnóstico papiloma invertido
- Lesión de configuración invertida
- Revestimiento por epitelio normal
- Uniformidad de las células epiteliales de revestimiento
- Mitosis ausentes o raras
- Formación de microquistes (criptas)
- Presencia de metaplasia escamosa




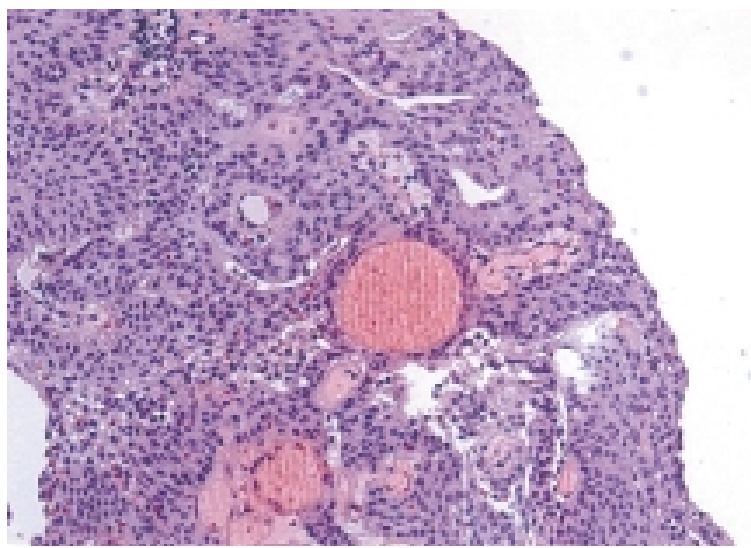

FIGURA 2. Neoplasia polipoide de células uroteliales formando cordones en el corion con raros microquistes (Hematoxilina-eosina).

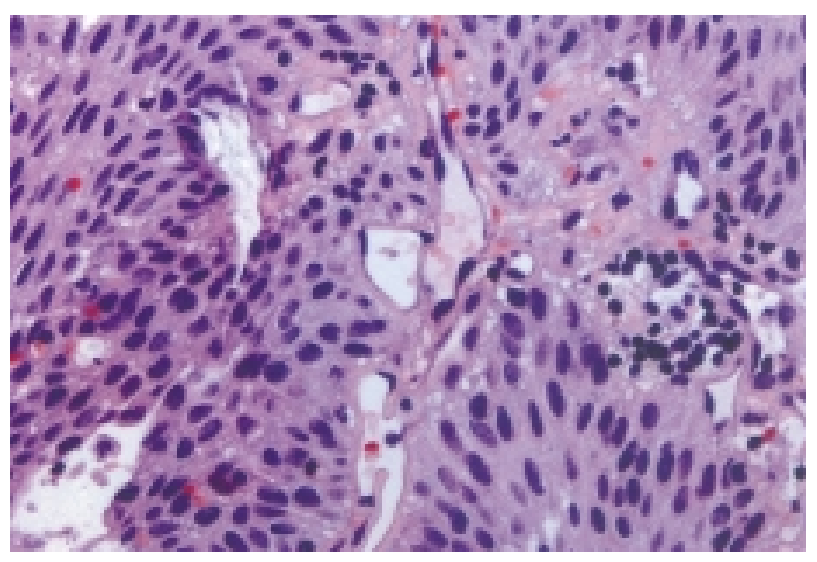

FIGURA 3. A mayor ampliación, mostrando urotelio normal en superficie (a la derecha) y células neoplásicas subyacentes con atipia minima (Hematoxilina-eosina).

zada en la periferia de la lesión. En la región central pueden verse quistes o criptas comunicantes con contenido de material PAS positivo, dándole un aspecto pseudoglandular adenomatoso. A pesar de la existencia de criterios histológicos bien establecidos, el diagnóstico diferencial con otras entidades es muchas veces difícil, especialmente con situaciones de cistitis quistica, cistitis glandular, nidos de von Brunn o incluso carcinoma urotelial de bajo grado. Debemos referir que en dos de los casos presentados coexistían en la misma pieza operatoria lesiones de este tipo, en uno de los casos lesiones de cistitis glandular y en el otro nidos de von Brunn.

Kunze describió posteriormente dos tipos básicos de papilomas invertidos de acuerdo con el patrón de crecimiento a los cuales llamó glandular y trabecular, dándoles patogénesis y pro- nóstico diferentes, aspectos que no se encuentran bien establecidos ${ }^{1,7,10}$. Hay igualmente tentativas para definir una clasificación histopatológica para estas lesiones aunque ninguna de ellas se encuentre ampliamente en uso ${ }^{8,11,12}$.

La etiología de los papilomas invertidos continua desconocida. En la descripción inicial Potts y Hirst sugirieron que estas lesiones resultarian de la transformación neoplásica de las células basales de las glándulas subtrigonales de Home o glándulas subcervicales de Albarrán ${ }^{2,8}$. Una vez que estas lesiones fueron posteriormente descritas en localizaciones extra-vesicales, esta hipótesis etiológica fue abandonada. Cummings y Matz defendieron la hipótesis de que el papiloma invertido no se trataría de una lesión propiamente neoplásica, siendo en realidad una reacción de tipo hiperplásico en respuesta a la inflamación crónica o agentes irritativos ${ }^{12-14}$. Esta misma hipótesis encuentra apoyo en el hecho de que estas lesiones se localizan predominantemente en áreas de mayor potencial de irritación crónica, son raramente multicentricas y presentan un indice de recurrencia muy bajo. Kunze y asociados lanzan por su parte la hipótesis de que los dos tipos de papilomas invertidos (trabecular y glandular) tengan origen distinta. De acuerdo con estos autores el tipo trabecular será originado a partir de alteraciones proliferativas de las células basales. El papiloma de tipo glandular sería originado de un proceso multifasico. Este se iniciaría con el desenvolvimiento de nidos de von Brunn. Estos evolucionarían para lesiones de cistitis quistica, cistitis glandular y finalmente terminarían en el propio papiloma ${ }^{1,8,9}$. Esta visión es igualmente llamativa vista la gran semejanza entre las lesiones de cistitis glandular y los papilomas, o incluso situaciones en que estas lesiones coexisten. La mayoría de los autores hoy en dia defienden que estas lesiones corresponden a una verdadera neoplasia, aunque de bajo grado de agresividad.

Otro aspecto que igualmente no está clarificado es el carácter benigno o maligno de estas lesiones. Por un lado, los aspectos histológicos, la baja tasa de recurrencia cuando comparada con la de los carcinomas uroteliales $(5 \%$ frente a cerca de $60 \%$ ), multifocalidad poco frecuente (próximo de los 5\%), ausencia de comportamiento invasivo y 
ausencia de metastización hablan a favor da benignidad. Por otro lado está bien establecida la relación de estas lesiones con el carcinoma urotelial. Este es relatado en las diversas series como ocurriendo de forma sincrónica o diferida en cerca de $10 \%$ de los casos, llegando a valores mas elevados para los papilomas del excretor alto. Hay incluso un caso descrito de coexistencia de estas lesiones en la misma pieza de resección transuretral $^{12}$. Estos datos sugieren que los factores envueltos en la génesis de estas lesiones son similares o comunes. En la serie presentada en un de los casos, tres meses después del diagnóstico de papiloma invertido fue verificado el aparecimiento de neoplasia de células de transición con características de invasividad. En los otros casos, a pesar de períodos de control prolongados (llegando a los 96 meses), no hubo evidencia de recidiva de las lesiones o desarrollo posterior de carcinoma transicional.

Por otro lado, las tentativas de identificar la agresividad potencial de estas lesiones o la probabilidad de desarrollo subsecuente de carcinoma transicional con determinación de las alteraciones nucleares e celulares, actividad proliferativa (expresión del antígeno nuclear Ki-67) y estudio de la proteína p53 presentan resultados dispares ${ }^{10,15}$.

El tratamiento de elección para el papiloma invertido es la resección endoscópica ${ }^{17}$. Para las lesiones del aparato excretor alto, el diagnóstico en la mayoría de los casos fué realizado de forma retrospectiva tras procedimientos ablativos (nefroureterectomia), dada la dificultad de diagnóstico preoperatorio. La instilación de citostáticos no está indicada, excepto lógicamente para los casos en que la coexistencia de tumor urotelial la indique.

\section{REFERENCIAS}

1. KUNZE E, SCHAUER A, SCHMITT M.: Histology and histogenesis of two different types of inverted papillomas. Cancer 1983; 51: 348.

2. POTTS AF, HIRST E.: Inverted papilloma of the bladder. J Urol 1963; 90: 175.

3. PASCHKIS R.: Uber adenome der Harnblase. Z Urol Chir 1927; 21: 315.

4. FERNÁNDEZ BORREL A, PEINADO IBARRA F, GÓMEZ-SANCHA F et al.: Cuatro nuevos casos de papiloma invertido urotelial. Actas Urol Esp 1998; 22 (7): 620-623.
5. HENDERSON DW, ALLEN PW, BOURNE AJ.: Inverted urinary papilloma: report of five cases and review of the literature. Virchows Arch A Path Anat Hist 1975; 366: 177.

6. ANDERSON C, JOHANSSON S, PETTERSON S.: Inverted papilloma of the urinary tract. $J$ Urol 1982; 127: 1132-1134.

7. SPEVACK L, HERSCHORN S, SRIGLEY J.: Inverted papilloma of the upper urinary tract. $J$ Urol 1995; 153: 1202-1204.

8. WITJES JA, BALKEN MR, VAN DE KAA CA.: The prognostic value of a primary inverted papilloma of the urinary tract. J Urol 1997; 158: 1500-1505.

9. RENFER LG, KELLEY J, BELVILLE WD.: Inverted papilloma of the urinary tract: histogenesis, recurrence and associated malignancy. J Urol 1988; 140: 832-834.

10. VALERO JA, REDONDO E, JIMÉNEZ C, GÓMEZ ML, MARTÍNEZ SL.: Papiloma invertido transicional: expressión del antígeno nuclear Ki-67 como factor pronóstico. Arch Esp Urol 1995; 48 (9): 887 892.

11. KIMURA G, TSUBOI N, NAKAJIMA H, YOSHIDA K, MASUGI Y, AKIMOTO M.: Inverted papilloma of the ureter with malignant transformation: a case report and review of the literature. The importance of the recognition of the inverted papillary tumour of the ureter. Urol Int 1987; 42: 30.

12. GRAINGER R, GIKAS PW, GROSSMAN HB.: Urothelial carcinoma occurring within an inverted papilloma of the ureter. $J$ Urol 1990; 143: 802-804.

13. MATZ LR, WISHART VA, GOODMAN MA.: Inverted urothelial papilloma. Pathology 1974; 6: 37.

14. CUMMINGS R.: Inverted papilloma of the bladder. $J$ Path 1974; 112: 225.

15. CHEVILLE JC, WU K, SEBO TJ, CHENG L, RIEHLE D, LOHSE CM, PANKRATZ VS.: Inverted urothelial papilloma- is ploidy, MIB-1 proliferative activity or p 53 protein accumulation predictive of urothelial carcinoma? Cancer 2000; 88: 632-636.

16. ISAAC J, LOWICHIK A, CARTWRIGHT P, ROHR R.: Inverted papilloma of the urinary bladder in children: case report and review of prognostic significance and biological potential behavior. J Ped Surg 2000; 35 (10): 1514-1516.

17. YIAGI H, IGAWA M, SHIINA H, SHIGENO K, YONEDA T, WADA Y, URAKAMI S.: Inverted papilloma of the urinary bladder in a girl. Urol Int 1999; 63 (4): 258-260.

\section{Dr. L. Xambre}

Serviço de Urologia

Hospital Pedro Hispano

Rua Dr. Eduardo Torres

4464-509 Matosinhos (Portugal)

e-mail: xambreluis@yahoo.com

(Trabajo recibido el 18 octubre de 2002) 